\title{
Polymerase chain reaction for detection of mucosal cytomegalovirus infection in patients with acute ulcerative colitis
}

\author{
Gloria S.Z. Tun, Mohammad Raza, Melissa F. Hale, Alan J. Lobo \\ Sheffield Teaching Hospital NHS Foundation Trust, United Kingdom
}

Abstract

Background Cytomegalovirus (CMV) infection is associated with acute exacerbations of ulcerative colitis (UC) but its clinical relevance remains uncertain. The primary aim of this study was to assess the prevalence of CMV infection in UC patients using viral polymerase chain reaction (PCR) analysis of mucosal biopsy samples. Secondary aims were to establish whether the disease was due to a primary infection or reactivation and to note associated risk factors and clinical outcomes.

Methods Since 2011, a policy of biopsy for CMV infection was adopted for severe UC patients in a large tertiary center. A retrospective review was undertaken to identify patients with mucosal biopsies for exacerbations of UC from October 2011 through January 2014.

Results Sixty biopsies for CMV PCR were obtained from 52 patients, 15 of whom were positive. In these patients, 9/9 tested were seropositive for anti-CMV IgG, while none were seropositive for anti-CMV IgM. Steroid refractory disease was a significant predictor of CMV positivity; however, there was no difference between the CMV-positive and -negative groups in rates of immunosuppression, or clinical and endoscopic severity. Six patients in the CMVpositive group received infliximab; all received concurrent antiviral therapy and did not require surgery.

Conclusions PCR of mucosal biopsies detected CMV infection due to viral reactivation in almost a third of patients with deteriorating or acute severe UC. Steroid refractory disease was significantly associated with CMV positivity, but no significant relationship was demonstrated with either disease severity or immunosuppression in our cohort. Treatment with anti-tumor necrosis factor agents was administered safely in combination with antiviral drugs.

Keywords Ulcerative colitis, cytomegalovirus, endoscopy, microbiology, pathology

Ann Gastroenterol 2019; 32 (1): 1-7

\section{Introduction}

Cytomegalovirus (CMV) infection may be a factor in acute exacerbations of ulcerative colitis (UC) [1-4].

Gastroenterology Department, Sheffield Teaching Hospital NHS Foundation Trust, United Kingdom

Conflict of Interest: None

Correspondence to: Dr Gloria S.Z. Tun, P1 ward, P floor, Royal Hallamshire Hospital, Glossop Road, Sheffield S10 2JF, United Kingdom, e-mail: Gloria.tun@sth.nhs.uk

Conference presentation: British Society of Gastroenterology Annual meeting 2014, Manchester

Received 22 March 2018; accepted 10 September 2018; published online 3 October 2018

DOI: https://doi.org/10.20524/aog.2018.0318
Laboratory evidence of past infection is found in $37-97 \%$ of the adult population $[5,6]$; following the primary infection, CMV persists in a state of lifelong latency. Patients with UC may be at increased risk of reactivation of latent CMV due to colonic inflammation and immunosuppression [7,8]. It has been suggested that, if mucosal CMV infection complicates an acute flare up of UC, outcomes are worse, with greater mortality and morbidity, and up to a $50 \%$ chance of colectomy [2-4,8].

The primary aim of this study was to assess the prevalence of CMV infection in patients with acute UC using viral polymerase chain reaction (PCR) analysis of mucosal biopsies. Secondary aims were: a) to clarify whether the presence of colonic CMV was due to viral reactivation or primary infection; b) to assess risk factors for CMV infection; and c) to assess the outcomes of the patients with CMV infection; in particular, those receiving anti-tumor necrosis factor (TNF)- $\alpha$ therapy. 


\section{Patients and methods}

\section{Patients}

Following the introduction of a policy of assessment for $\mathrm{CMV}$ infection, patients undergoing lower gastrointestinal endoscopy with mucosal biopsies for exacerbations of UC were identified retrospectively from laboratory records. Adult patients (aged $\geq 16$ ) who had a biopsy from October 2011 through January 2014 were included. The diagnosis of UC was based on histological criteria [9]. Extent of disease was recorded using the Montreal classification [10].

Clinical, endoscopic, laboratory and histological data were retrieved from patients' clinical notes and hospital electronic systems. The Mayo disease activity score [11] within $24 \mathrm{~h}$ of endoscopy was calculated retrospectively. Baron score [12] was used to describe endoscopic severity.

Systemic corticosteroid use was assessed as any steroid exposure within 1 month, cumulative steroid dose and significant steroid exposure (defined as prednisolone $\geq 10 \mathrm{mg} /$ day for $\geq 14$ days prior to biopsy). Prednisolone equivalent dose was calculated when patients had received intravenous hydrocortisone, with $20 \mathrm{mg}$ hydrocortisone being equivalent to $5 \mathrm{mg}$ prednisolone [13].

Patients were deemed to be refractory to systemic corticosteroid therapy if they needed escalation of treatment to anti-TNF therapy, cyclosporine or surgery, or had a C-reactive protein (CRP) level $>35 \mathrm{mg} / \mathrm{L}$ on day 3 of in-patient treatment with intravenous corticosteroids. Clinical response was established using physician global assessment based on a clear decrease in stool frequency and CRP.

\section{CMV infection}

The presence of CMV infection was assessed using a combination of PCR of viral DNA from mucosal biopsies, PCR of viral DNA from peripheral blood samples, serology for antibodies to CMV and histopathological assessment of mucosal biopsy samples.

\section{Mucosal and peripheral blood PCR}

Biopsy samples were taken with standard endoscopy forceps (Boston Scientific $2.8 \mathrm{~mm}$ ) and transported in saline. Nucleic acid was extracted from biopsies using Qiagen Qiamp DNA Mini kit (Hilden, Germany) and amplified using Qiagen Artus CMV kit on the Qiagen Rotor-gene (Qiagen, Hilden, Germany). For peripheral blood, plasma samples were extracted using a magnetic particle-based extraction assay, MagNA Pure 96 system (Roche, Basel, Switzerland). The purified nucleic acid was then tested using the same PCR kit as the biopsies (Qiagen, Hilden, Germany). CMV DNA testing was performed in a United Kingdom Accreditation Service (UKAS) laboratory.

\section{Serology}

CMV IgG and IgM antibody detection was performed by chemiluminescence using a Liaison ${ }^{\circ} \mathrm{XL}$ automated analyzer (DiaSorin, Saluggia, Italy).

\section{Histology}

Biopsy tissue was fixed in formalin, processed using alcohol, xylene and wax and then cut into sections. All samples were stained with hematoxylin and eosin (H\&E) and were examined using light microscopy and reported by a consultant histopathologist. Where additional immunohistochemical (IHC) assessment was undertaken, this involved an additional stain of monoclonal antibodies specific to CMV antigens.

\section{Other tests}

\section{CRP}

Plasma CRP levels were measured with a particle enhanced immunoturbidimetric assay, Roche/Hitachi Cobas c 501 (Roche, Basel, Switzerland).

\section{Statistical analysis}

The Mann-Whitney $U$ test was used to compare the Baron and Mayo scores between the biopsy CMV PCRpositive and -negative patients. A binomial logistic regression model was used for analysis of possible predictors of CMV positivity. Chi-square analysis was used to compare immunosuppression, rates of steroid refractory disease and surgery between CMV PCR biopsy-positive and -negative patients. IBM SPSS statistics and Microsoft Excel were used. A P value $<0.05$ and $95 \%$ confidence intervals were deemed statistically significant.

\section{Ethical statement}

Ethical approval was not required as the review was a retrospective service evaluation of routine clinical care.

\section{Results}

\section{Clinical and demographic characteristics}

Fifty-two patients were identified and altogether 65 endoscopies were performed. Biopsies for CMV PCR were taken from 60 of these. Baseline clinical and demographic characteristics of all patients are shown in Table 1. Twelve of the 52 patients underwent repeat endoscopy and 1 patient 
underwent 3 endoscopies during admission. Patients underwent repeat endoscopy for ongoing clinical symptoms despite treatment, manifested as a raised Mayo score or ongoing stool frequency. Further details of the patients undergoing repeat endoscopy are shown in Table 2. Of the 15 CMV-positive patients, 10 had mucosal CMV DNA present on the biopsies from their first endoscopy and did not have a repeat endoscopy.

\section{CMV infection}

Table 3 summarizes the findings for CMV infection. Plasma CMV DNA PCR was performed in 11/15 CMV biopsy-positive patients and in 9/37 CMV biopsy-negative patients. Plasma CMV DNA PCR was positive in 6/11 CMV biopsy-positive patients and $0 / 9$ negative patients. Therefore, in these patients, the sensitivity was $55 \%$ and specificity $100 \%$, using the presence of biopsy CMV DNA as the standard.

Table 1 Baseline clinical and demographic characteristics of patients

\begin{tabular}{lcc}
\hline Patient characteristics & $\begin{array}{c}\text { Mucosal CMV DNA } \\
\text { positive (n=15) }\end{array}$ & $\begin{array}{c}\text { Mucosal CMV DNA } \\
\text { negative }(\mathrm{n}=37)\end{array}$ \\
\hline Gender M: F & $9: 6$ & $14: 23$ \\
Age in years, mean (range) & $39.4(18-75)$ & $39.6(18-85)$ \\
Duration of disease in months, mean (range) & $45.4(0-192)$ & $81.1(0-420)$ \\
Extent of disease: Montreal Classification: & & $5(14 \%)$ \\
E1 & 0 & $12(32 \%)$ \\
E2 & $5(33 \%)$ & $19(51 \%)$ \\
E3 & $7(47.7 \%)$ & $1(3 \%)$ \\
Unknown & $3(20 \%)$ & $5(14 \%)$ \\
Medication: & $6(40 \%)$ & $12(32 \%)$ \\
Topical therapy (5-aminosalicylate) & $5(33 \%)$ & 0 \\
Thiopurines & 0 & $23(62 \%)$ \\
Cyclosporine & $10(67 \%)$ & $4(11 \%)$ \\
Steroids & $3(20 \%)$ & $9(6-12)$ \\
Anti-tumor necrosis factor- $\alpha$ & $9(3-12)$ & $2(1-3)$ \\
Mayo score, median (range) & $3(1-3)$ & \\
Baron score, median (range) & & \\
\hline
\end{tabular}

CMV, cytomegalovirus; DNA, deoxyribonucleic acid

Table 2 Timing, Mayo score and mucosal CMV PCR results of patients undergoing repeat endoscopy

\begin{tabular}{|c|c|c|c|c|c|c|c|c|c|}
\hline \multirow{2}{*}{$\begin{array}{l}\text { Patient } \\
\text { Mucosal } \\
\text { CMV DNA } \\
\text { status }\end{array}$} & \multicolumn{3}{|c|}{ First endoscopy } & \multicolumn{3}{|c|}{ Second endoscopy } & \multicolumn{3}{|c|}{ Third endoscopy } \\
\hline & $\begin{array}{l}\text { Day of } \\
\text { endoscopy }\end{array}$ & $\begin{array}{l}\text { Mayo } \\
\text { score }\end{array}$ & $\begin{array}{l}\text { Mucosal } \\
\text { CMV DNA } \\
\text { PCR results }\end{array}$ & $\begin{array}{l}\text { Day of } \\
\text { endoscopy }\end{array}$ & $\begin{array}{l}\text { Mayo } \\
\text { score }\end{array}$ & $\begin{array}{l}\text { Mucosal } \\
\text { CMV DNA } \\
\text { PCR results }\end{array}$ & $\begin{array}{l}\text { Day of } \\
\text { endoscopy }\end{array}$ & $\begin{array}{l}\text { Mayo } \\
\text { score }\end{array}$ & $\begin{array}{l}\text { Mucosal CMV } \\
\text { DNA PCR } \\
\text { results }\end{array}$ \\
\hline Positive & Day 3 & 10 & Not done & Day 13 & 6 & 63900 & & & \\
\hline Positive & Day 1 & 12 & Negative & Day 6 & 10 & 776 & & & \\
\hline Positive & Day 10 & 11 & Not done & Day 19 & 5 & 94700 & & & \\
\hline Positive & Day 0 & 12 & Negative & Day 10 & 5 & 5360 & & & \\
\hline Positive & Day 3 & 11 & Negative & Day 10 & 10 & 29300 & Day 21 & 9 & Negative \\
\hline Negative & Day 2 & 9 & Negative & Day 9 & 5 & Not done & & & \\
\hline Negative & Day 8 & 9 & Not done & Day 17 & 9 & Negative & & & \\
\hline Negative & Day 0 & 9 & Negative & Day 10 & 7 & Negative & & & \\
\hline Negative & Day 1 & 9 & Negative & Day 7 & 7 & Negative & & & \\
\hline Negative & Day 1 & 12 & Negative & Day 7 & 10 & Negative & & & \\
\hline Negative & Day 1 & 10 & Negative & Day 6 & 6 & Negative & & & \\
\hline Negative & Day 1 & 12 & Negative & Day 17 & 10 & Negative & & & \\
\hline
\end{tabular}

Day of endoscopy signifies day of inpatient stay

CMV, cytomegalovirus; $P C R$, polymerase chain reaction; DNA, deoxyribonucleic acid 
Histological analysis using H\&E was performed in 60/65 biopsies. Four of the remaining 5 biopsies did not have an associated histological assessment as they were repeat endoscopies to specifically assess for biopsy CMV PCR. One patient in the CMV biopsy-negative group did not have any histological assessment. IHC analysis was performed in 7 patients and was negative in all. In the 4 CMV PCR biopsypositive patients who had IHC performed, the corresponding biopsy DNA PCR levels were <1000, 63,900, 89,200, and $1,540,000$ copies/ $\mathrm{mL}$ of the extract.

\section{Predictors of CMV positivity}

Table 4 shows the results of a binomial logistic regression model in assessing variables for a relationship to CMV positivity on biopsy PCR. The use of topical 5-aminosalicylate therapy and steroid refractory disease were significantly associated with CMV positivity $(\mathrm{P}=0.03$ and $\mathrm{P}=0.04$, respectively) and being male showed near significance $(\mathrm{P}=0.05)$.

\section{Clinical and endoscopic severity}

Clinical and endoscopic severity did not differ between CMV biopsy-positive and -negative groups. For the biopsy PCR-positive group, median Mayo score was 9 (range 3-12) and the median Baron score was 3 (range 1-3), while in the biopsy PCR-negative group, median Mayo score was also 9 (range 6-12) and median Baron score 2 (range 1-3) $(\mathrm{P}=0.09$ and 0.81, respectively, for Mayo and Baron scores).

Biopsy CMV DNA levels in relation to differing Mayo and Baron scores in biopsy-positive patients are shown in Fig. 1, with an outlier of 1,540,000 copies/mL displayed in red.

\section{Use of immunosuppressant medications}

There was no significant difference in corticosteroid use between biopsy CMV PCR-positive and -negative patients:

- 10/15 (66.7\%) CMV-positive patients had corticosteroid exposure compared with 22/37 (59.5\%) CMV-negative patients $(\mathrm{P}=0.62)$

- 3/15 (20\%) CMV-positive patients had received prednisolone $\geq 10 \mathrm{mg}$ /day for $\geq 14$ days prior to biopsy compared to $14 / 37$ (37.8\%) CMV-negative patients $(\mathrm{P}=0.21)$

- The median cumulative corticosteroid dose prior to biopsy was $0.70 \mathrm{~g}$ prednisolone equivalent for CMVpositive patients (range $0-4.87 \mathrm{~g}$ ) and $0.40 \mathrm{~g}$ prednisolone equivalent for CMV-negative patients (range 0-3.09 g) $(\mathrm{P}=0.08)$

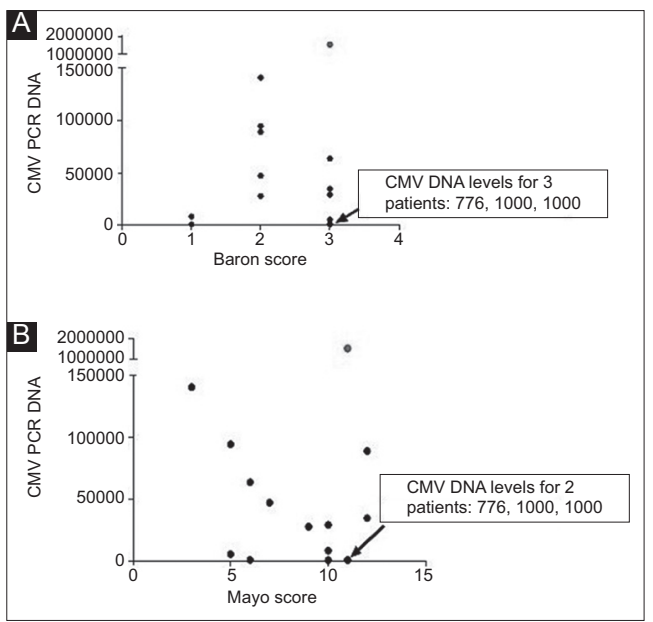

Figure 1 The relation of Baron (A) and Mayo (B) scores to mucosal CMV PCR DNA level in biopsy CMV-positive patients CMV, cytomegalovirus; PCR, polymerase chain reaction

Table 3 Summary of investigation findings for patients with positive mucosal CMV DNA

\begin{tabular}{llcc}
\hline Investigation & & Results & \\
\hline Mucosal CMV DNA PCR (copies/mL) & Median & DNA detected n=15 & DNA not detected n=37 \\
& Range & $29300<1000-1540000$ & 14 \\
Serology & CMV IgG positive & 9 & 11 \\
& CMV IgG negative & 0 & 12 \\
Serology & CMV IgG not tested & 6 & 0 \\
& CMV IgM positive & 0 & 26 \\
Plasma CMV PCR DNA & CMV IgM negative & 8 & 11 \\
& CMV IgM not tested & 7 & 0 \\
Histological assessment & Plasma CMV DNA detected & 6 \\
& Plasma CMV DNA not detected & 6 & 9 \\
& Plasma CMV DNA not tested & 4 & 28 \\
& CMV IHC tested & 4 & 3 \\
& CMV IHC not tested & 11 & 34 \\
& CMV IHC positive & 0 & 3 \\
\hline
\end{tabular}

CMV, cytomegalovirus; PCR, polymerase chain reaction; DNA, deoxyribonucleic acid; IgG, immunoglobulin G; IgM, immunoglobulin M; IHC, immunohistochemistry 
Table 4 Binomial logistic regression analysis of clinical variables and their impact on CMV positivity

\begin{tabular}{lc}
\hline Variable & $\begin{array}{c}\text { P-value }(95 \% \\
\text { confidence interval) }\end{array}$ \\
\hline Age & $0.40(0.973-1.071)$ \\
Sex (male) & $0.05(0.018-0.989)$ \\
\hline Duration of disease (months) & $0.12(0.978-1003)$ \\
\hline Treatment: & \\
\hline Topical therapy (5-ASA) & $0.03(1.285-81.554)$ \\
\hline Thiopurines & $0.35(0.079-2.476)$ \\
\hline Corticosteroid use & $0.38(0.051-3.127)$ \\
\hline Anti-TNF agents & $0.10(0.686-91.452)$ \\
\hline Mayo score & $0.52(0.514-1.397)$ \\
Baron score & $0.37(0.477-7.323)$ \\
\hline Steroid refractory disease & $0.04(1.164-90.171)$ \\
\hline
\end{tabular}

Steroid refractory disease and use of topical therapy were both significantly associated with CMV positivity

5-ASA, 5-aminosalicylate; TNF, tumor necrosis factor

There was also no significant difference between the groups in the use of thiopurines prior to biopsy: 5/15 (33\%) CMV PCR biopsy-positive patients and 12/37 (32\%) CMV PCR biopsynegative patients $(\mathrm{P}=0.95)$. Nor was there a difference in the prior use of anti-TNF agents, with 3/15 (20\%) CMV PCR biopsy-positive patients and 4/37 (11\%) CMV PCR biopsynegative patients receiving these agents $(\mathrm{P}=0.38)$.

Mucosal CMV PCR results were compared between biopsies on admission (day 0-1 after admission) or after in-patient treatment (day $2+$ after admission), as patients in the latter group would have received more days of intravenous hydrocortisone. Eight patients were excluded as they had their initial biopsy as an outpatient, leaving 52 endoscopies for comparison: of these, 6/25 (24\%) were CMV positive on admission and 7/27 (26\%) were CMV positive after inpatient treatment $(\mathrm{P}=0.87)$. The interval to endoscopy after inpatient treatment (day $2+$ group) ranged from 2-21 days, median 6 days.

\section{Antiviral therapy and clinical outcomes}

In the CMV-positive group, $14 / 15$ (93.3\%) were treated with antiviral medication and $11 / 14(78.6 \%)$ responded to treatment. Treatments received and outcomes are shown in Fig. 2. No patients experienced significant side-effects from the antiviral therapy. There was no significant difference in rates of steroid refractory disease $(10 / 15,66.7 \%$ in the CMV-positive group and $14 / 37,37.8 \%$ in the $\mathrm{CMV}$-negative group, $\mathrm{P}=0.06$ ) nor rates of surgery $(3 / 15,20 \%$ in the CMV-positive patients and $2 / 37,5.4 \%$ in the $C M V$-negative patients, $\mathrm{P}=0.11$ ).

\section{Anti-TNF agents}

In the CMV-positive group, 3/15 patients were receiving anti-TNF therapy on admission. All three had high levels of

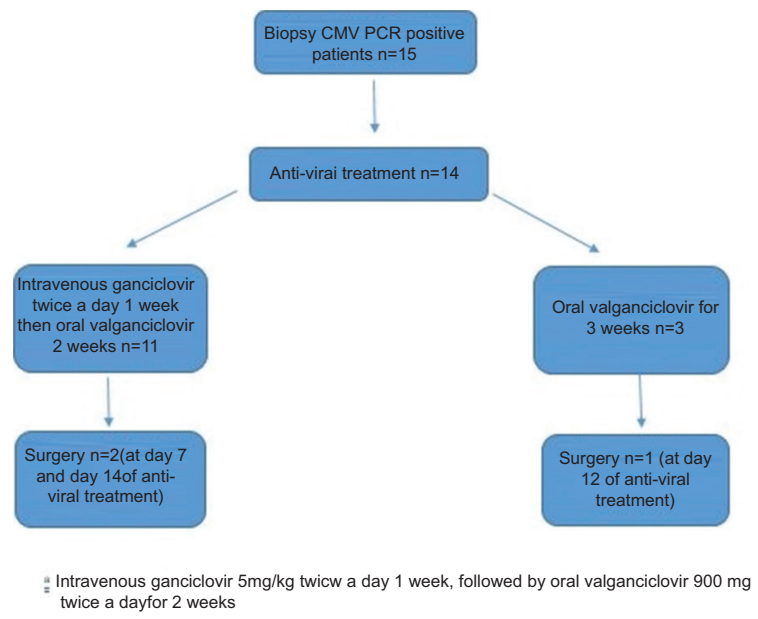

Figure 2 Antiviral treatment regimens and outcomes in biopsy CMVpositive patients $\mathrm{CMV}$, cytomegalovirus; $\mathrm{PCR}$, polymerase chain reaction

CMV DNA in their biopsies (34,900, 28,000, and 47,400 copies/ $\mathrm{mL}$ of the extract). During admission, a further 3 patients were escalated to anti-TNF therapy in the CMV-positive group. All 6 patients receiving infliximab (IFX) also received concomitant antiviral therapy. All responded to medical treatment and did not require escalation to surgery. In the CMV-negative group 3/27 were on anti-TNF therapy on admission and a further 9 were started on anti-TNF as treatment escalation. All 12 responded to medical treatment.

\section{Discussion}

This study demonstrated that nearly a third of patients with severe or deteriorating UC had concomitant mucosal CMV infection. This is higher than previously reported; the difference may be due to case-mix or the nature of the diagnostic test used in detecting mucosal CMV infection. It has been suggested that CMV disease in UC is due to reactivation of the dormant virus in myeloid progenitor and endothelial cells $[7,8,14,15]$. Our results would be consistent with this as serology showed an absence of primary infection, though reinfection remains a possibility.

Concerns remain about the lack of clinical specificity in using tissue PCR as a diagnostic tool $[7,15]$. Nevertheless, it allows for rapid results and compared to IHC it is more sensitive, with a high negative predictive value [16]. Although the different diagnostic methods vary in their ability to detect CMV infection, the presence of CMV DNA in the tissue, detected by PCR, indicates infection is present and that the $\mathrm{CMV}$ is not latent. In the latent state the virus would be residing in myeloid progenitor cells and is undetectable by IHC, H\&E, and PCR. The European Crohn's and Colitis Organisation (ECCO) recommend tissue PCR or IHC as the methods of choice for diagnosing CMV colitis [17]. Despite the high prevalence of PCR-detected infection, histopathology_albeit without routine IHC_did not detect any cases in our series. This discrepancy has also been noted by others $[1,7,18]$, as CMV inclusion bodies have a high specificity 
for CMV but may have a sensitivity as low as $20 \%$ [19]. Seven of our 52 patients had an IHC assessment and it is uncertain whether additional IHC, if performed for all the patients, would have improved the yield. However, this study did not aim to compare IHC with mucosal CMV PCR for the diagnosis of CMV colitis in UC. Instead, it highlights that in routine clinical practice additional IHC assessment may only be performed in a minority of cases, such as when inclusion bodies are seen on H\&E stain, to confirm the presence of CMV. However, using this practice, CMV disease may be missed because of the low sensitivity of inclusions bodies. Therefore, ideally either IHC or mucosal CMV PCR should be used for the diagnosis of CMV colitis in patients with acute exacerbations of UC.

The quantitative nature of PCR gives the potential for stratification of severity and may allow a therapeutic threshold for treatment-as, for example, in post-transplantation patients [20,21]. Roblin et al showed that a CMV DNA load $>250$ copies $/ \mathrm{mg}$ predicted resistance to three lines of treatment in UC [3], but the different units of quantification in our laboratory (copies $/ \mathrm{mL}$ ) preclude comparison with this study. Universal agreement regarding the units of measurement for CMV PCR would aid the generalizability of results and comparisons of findings from different studies. Higher DNA levels reflect increased viral load, but a positive correlation was not found between DNA level and clinical or endoscopic severity in our study. Variation in biopsy size is unlikely to account for this, as a minimal biopsy volume was required for assessment. However, it may be due to a non-uniform mucosal viral load, as higher levels of CMV DNA have been found in the base and edge of ulcers in resected UC specimens [22] and the location of the biopsy site was not documented in our cohort.

When independent variables were assessed as predictors of CMV positivity, steroid refractory disease was shown to be significant. Unexpectedly, no significant difference was present in rates of immunosuppression, systemic steroid use or thiopurines between CMV-negative and -positive patients. This contrasts with previous studies that suggested increased duration of steroid use, thiopurines and cyclosporine are associated with CMV reactivation [4,8,18,23-25]. No significant difference was shown with use of anti-TNF inhibitors, a finding that reinforces previous results [26,27]. Baseline characteristics of this cohort showed that those with positive mucosal CMV DNA had a much shorter duration of UC compared to those with negative CMV DNA (mean of 45 months compared to 81). The shorter duration of disease may indicate less controlled disease, as treatment may be "stepped up" compared to those with more established disease. Less well controlled disease would be associated with ongoing colonic inflammation, which would predispose to CMV reactivation and may explain why patients with positive mucosal CMV DNA had a shorter duration of disease. A limitation in our study is the sample size, as only 15/52 patients were biopsy positive for CMV DNA. However, to strengthen the statistical analyses, such as in the case of corticosteroid use, this was reviewed in three different ways: any corticosteroid exposure, prednisolone $\geq 10 \mathrm{mg} /$ day for $\geq 14$ days and median cumulative corticosteroid dose.

The uncertainty of whether antiviral treatment is beneficial in patients positive for CMV on mucosal PCR during a flare of UC remains. A meta-analysis concluded that the use of antivirals did not improve outcomes [28], but was limited by information on the antivirals used, matching for severity or steroid-refractory rates. Two studies included in the metaanalysis only used antivirals in patients with steroid refractory disease. Studies in post-transplant patients have utilized immune monitoring assays as a tool in assessing who would benefit from antiviral therapy [29,30]. These assays allow the measurement of a patient's T-cell response to CMV as a predictor of the patient's own ability to respond to the infection, indicating which patients may spontaneously clear the infection and which patients may need antiviral therapy $[29,30]$. However, these assays are not currently routinely used in clinical practice and current guidelines recommend a 2-3 week course of antiviral therapy [17]. Successful remission was achieved in nearly $80 \%$ of the CMV-positive group in our study following antiviral treatment, similar to previously reported remission rates following antiviral therapy in UC [15,28]. Our experience suggests that IFX, when used with concomitant antiviral therapy, is safe in treating refractory acute UC with associated mucosal CMV infection. This supports a recent study, where similar rates of remission with IFX were seen in both CMV-positive and -negative groups [31] and a stable or reduced colonic CMV DNA load was documented despite the use of IFX [31]. A randomized trial to demonstrate the benefit

\section{Summary Box}

\section{What is already known:}

- Cytomegalovirus (CMV) persists in a state of lifelong latency following infection, reported in up to $97 \%$ of the adult population

- Mucosal CMV infection can complicate an exacerbation of ulcerative colitis (UC) and has been associated with higher mortality and morbidity, including an increased risk of colectomy

- Guidelines recommend tissue polymerase chain reaction $(\mathrm{PCR})$ or immunohistochemical analysis as the methods of choice for diagnosing CMV colitis in UC

\section{What the new findings are:}

- Using mucosal PCR, CMV infection due to viral reactivation was diagnosed in nearly a third of patients with acute or deteriorating UC

- Steroid refractory disease was significantly associated with CMV positivity

- The use of antiviral therapy achieved remission in nearly $80 \%$ of the CMV-positive group

- Infliximab, when used with concomitant antiviral therapy, was safe in treating refractory acute UC with associated mucosal CMV infection 
of antiviral therapy would need to utilize a design that avoided inappropriate delay before escalating to medical treatment or surgery. Larger, prospective studies using tissue PCR are now needed to clarify the risk factors for CMV infection, optimize methods to allow comparison across centers, and to investigate the role of the immune monitoring assays in UC, with a view to a randomized trial of antiviral treatment.

In conclusion, mucosalCMV infection dueto viral reactivation is common in acute or deteriorating UC. Concomitant CMV infection should be considered in the management of these patients, especially in those who are steroid refractory. Mucosal PCR is sensitive, rapid and has a high negative predictive value in the diagnosis of CMV infection in UC patients. In our cohort, the use of antivirals achieved remission in nearly $80 \%$ of the CMV-positive group and IFX, when used with concomitant antiviral therapy, was safe in treating refractory acute UC with associated mucosal CMV infection.

\section{References}

1. Yoshino T, Nakase H, Ueno S, et al. Usefulness of quantitative realtime PCR assay for early detection of cytomegalovirus infection in patients with ulcerative colitis refractory to immunosuppressive therapies. Inflamm Bowel Dis 2007;13:1516-1521.

2. Ayre K, Warren BF, Jeffery K, Travis SP. The role of CMV in steroidresistant ulcerative colitis: A systematic review. J Crohns Colitis 2009;3:141-148.

3. Roblin X, Pillet S, Oussalah A, et al. Cytomegalovirus load in inflamed intestinal tissue is predictive of resistance to immunosuppressive therapy in ulcerative colitis. Am J Gastroenterol 2011;106:2001-2008.

4. Kishore J, Ghoshal U, Ghoshal UC, et al. Infection with cytomegalovirus in patients with inflammatory bowel disease: prevalence, clinical significance and outcome. J Med Microbiol 2004;53:1155-1160.

5. Njeru DG, Mwanda WO, Kitonyi GW, Njagi EC. Prevalence of cytomegalovirus antibodies in blood donors at the National Blood Transfusion Centre, Nairobi. East Afr Med J 2009;86:S58-S61.

6. Zhang LJ, Hanff P, Rutherford C, Churchill WH, Crumpacker CS. Detection of human cytomegalovirus DNA, RNA, and antibody in normal donor blood. J Infect Dis 1995;171:1002-1006.

7. Lawlor G, Moss AC. Cytomegalovirus in inflammatory bowel disease: pathogen or innocent bystander? Inflamm Bowel Dis 2010;16:1620-1627.

8. Nguyen M, Bradford K, Zhang X, Shih DQ. Cytomegalovirus reactivation in ulcerative colitis patients. Ulcers 2011;2011:282507.

9. Jenkins D, Balsitis M, Gallivan S, et al. Guidelines for the initial biopsy diagnosis of suspected chronic idiopathic inflammatory bowel disease. The British Society of Gastroenterology Initiative. J Clin Pathol 1997;50:93-105.

10. Satsangi J, Silverberg MS, Vermeire S, Colombel JF. The Montreal classification of inflammatory bowel disease: controversies, consensus, and implications. Gut 2006;55:749-753.

11. Rutgeerts P, Sandborn WJ, Feagan BG, et al. Infliximab for induction and maintenance therapy for ulcerative colitis. $\mathrm{N} \mathrm{Engl} \mathrm{J}$ Med 2005;353:2462-2476.

12. Baron JH, Connell AM, Lennard-Jones JE. Variation between observers in describing mucosal appearances in proctocolitis. $\mathrm{Br}$ Med J 1964;1:89-92.

13. Joint Formulary Committee. British National Formulary (online) London: BMJ Group and Pharmaceutical Press. Glucocorticoid therapy. Available from: https://bnf.nice.org.uk/treatmentsummary/glucocorticoid-therapy.html [Accessed on 27/09/18].

14. Pillet S, Pozzetto B, Jarlot C, Paul S, Roblin X. Management of cytomegalovirus infection in inflammatory bowel diseases. Dig Liver Dis 2012;44:541-548.

15. Sager K, Alam S, Bond A, Chinnappan L, Probert CS. Review article: cytomegalovirus and inflammatory bowel disease. Aliment Pharmacol Ther 2015;41:725-733.

16. Weinberg A, Hodges TN, Li S, Cai G, Zamora MR. Comparison of PCR, antigenemia assay, and rapid blood culture for detection and prevention of cytomegalovirus disease after lung transplantation. J Clin Microbiol 2000;38:768-772.

17. Rahier JF, Magro F, Abreu C, et al; European Crohn's and Colitis Organisation (ECCO). Second European evidence-based consensus on the prevention, diagnosis and management of opportunistic infections in inflammatory bowel disease. J Crohns Colitis 2014;8:443-468.

18. Domènech E, Vega R, Ojanguren I, et al. Cytomegalovirus infection in ulcerative colitis: a prospective, comparative study on prevalence and diagnostic strategy. Inflamm Bowel Dis 2008;14:1373-1379.

19. Mattes FM, McLaughlin JE, Emery VC, Clark DA, Griffiths PD. Histopathological detection of owl's eye inclusions is still specific for cytomegalovirus in the era of human herpesviruses 6 and 7 . J Clin Pathol 2000;53:612-614.

20. Sanghavi SK, Abu-Elmagd K, Keightley MC, et al. Relationship of cytomegalovirus load assessed by real-time PCR to pp65 antigenemia in organ transplant recipients. J Clin Virol 2008;42:335-342.

21. Gerna G, Lilleri D. Monitoring transplant patients for human cytomegalovirus: diagnostic update. Herpes 2006;13:4-11.

22. Zidar N, Ferkolj I, Tepeš K, et al. Diagnosing cytomegalovirus in patients with inflammatory bowel disease-by immunohistochemistry or polymerase chain reaction? Virchows Arch 2015;466:533-539.

23. Cottone M, Pietrosi G, Martorana G, et al. Prevalence of cytomegalovirus infection in severe refractory ulcerative and Crohn's colitis. Am J Gastroenterol 2001;96:773-775.

24. Minami M, Ohta M, Ohkura T, et al. Cytomegalovirus infection in severe ulcerative colitis patients undergoing continuous intravenous cyclosporine treatment in Japan. World J Gastroenterol 2007; 13:754-760.

25. Pickering $O$, Weinstein $T$, Rubin LG. Fatal disseminated cytomegalovirus infection associated with infliximab and 6-mercaptopurine therapy in a child with Crohn disease. Pediatr Infect Dis J 2009;28:556.

26. Criscuoli V, Mocciaro F, Orlando A, Rizzuto MR, Renda MC, Cottone M. Cytomegalovirus disappearance after treatment for refractory ulcerative colitis in 2 patients treated with infliximab and 1 patient with leukapheresis. Inflamm Bowel Dis 2009;15:810-811.

27. Nakase H, Chiba T. TNF-alpha is an important pathogenic factor contributing to reactivation of cytomegalovirus in inflamed mucosa of colon in patients with ulcerative colitis: lesson from clinical experience. Inflamm Bowel Dis 2010;16:550-551.

28. Kopylov U, Eliakim-Raz N, Szilagy A, Seidman E, Ben-Horin S, Katz L. Antiviral therapy in cytomegalovirus-positive ulcerative colitis: a systematic review and meta-analysis. World J Gastroenterol 2014;20:2695-2703.

29. Fernández-Ruiz M, Kumar D, Humar A. Clinical immunemonitoring strategies for predicting infection risk in solid organ transplantation. Clin Transl Immunology 2014;3:e12.

30. Lisboa LF, Kumar D, Wilson LE, Humar A. Clinical utility of cytomegalovirus cell-mediated immunity in transplant recipients with cytomegalovirus viremia. Transplantation 2012;93:195-200.

31. Pillet $S$, Jarlot $C$, Courault $M$, et al. Infliximab does not worsen outcomes during flare-ups associated with cytomegalovirus infection in patients with ulcerative colitis. Inflamm Bowel Dis 2015;21:1580-1586. 